\title{
Intelligent Autofeedback and Safety Early-Warning for Underground Cavern Engineering during Construction Based on BP Neural Network and FEM
}

\author{
Lei Xu, ${ }^{1}$ Taijun Zhang, ${ }^{2}$ and Qingwen $\operatorname{Ren}^{3}$ \\ ${ }^{1}$ College of Water Conservancy and Hydropower Engineering, Hohai University, Nanjing 210098, China \\ ${ }^{2}$ Zaozhuang City Water Survey and Design Institute, Zaozhuang 277800, China \\ ${ }^{3}$ Department of Mechanics, Hohai University, Nanjing 210098, China
}

Correspondence should be addressed to Lei Xu; leixu@hhu.edu.cn

Received 23 August 2014; Accepted 12 October 2014

Academic Editor: Song Cen

Copyright (C) 2015 Lei Xu et al. This is an open access article distributed under the Creative Commons Attribution License, which permits unrestricted use, distribution, and reproduction in any medium, provided the original work is properly cited.

The low efficiency of feedback analysis is one of the main shortcomings in the construction of underground cavern engineering. With this in mind, a method of intelligent autofeedback and safety early-warning for underground cavern is proposed based on BP neural network and FEM. The training sample points are chosen by using uniform test design method, and the autogeneration of FEM calculation file for ABAQUS is realized by using the technique of file partition, information grouping, and orderly numbering. Then, intelligent autoinversion of mechanics parameters is realized, and the automatic connection of parameter inversion, subsequent prediction, and safety early-warning is achieved. The software of intelligent autofeedback and safety earlywarning for underground cavern engineering during construction is developed. Finally, the applicability of the proposed method and the developed software is verified through an application example of underground cavern of a pumped-storage power station located in Southwest China.

\section{Introduction}

The development of civil engineering, water conservancy and hydropower, transportation engineering, and mining industry boosts continuous and fast construction of underground cavern in China. Underground caverns are often constructed in rock masses with complicated geological environment and mechanical properties, so their design and construction are usually faced with limited and inaccurate data such as mechanical parameters of surrounding rock. As a result, engineering analogy method or experience judgment method has been playing an important role in the design and construction of underground cavern. However, with the rapid development of engineering computational methods, monitoring technique, and artificial intelligence method, feedback analysis during construction [1-4] has also become a more and more important method in the design and construction of underground cavern. The inversion of mechanical parameters, prediction of the stress and deformation of the surrounding rock in the subsequent construction steps, and safety early-warning are main procedures of feedback analysis. In recent years, scholars in this field have done many valuable research works [5-9], but there are still some shortcomings. From the viewpoint of practical application, the main shortcomings are that the efficiency of the inversion of mechanical parameters is low and the exchange of data among parameter inversion, subsequent prediction, and safety early-warning cannot be conducted automatically.

In general, there are mainly two methods which can be used to inverse the mechanical parameters of surrounding rock. One is direct inversion method, and the other is indirect inversion method. As to direct inversion method, the optimization inversion model, which takes the inversion parameters as design variables, should be firstly established based on the monitoring data. Then, the inversion of mechanical parameters of surrounding rock can be accomplished based on a certain optimization method. As to indirect inversion method, the mapping model, which is used to simulate the relation between the inversion parameters and 
monitoring data, should be firstly established by making use of artificial neural network or machine learning method. Then, the inversion parameters can be obtained by inputting the monitoring data into the mapping model. Since indirect inversion method does not require iterative calculation, it is commonly regarded that the efficiency of indirect inversion method is higher than that of direct inversion method. Owing to this point of view, the indirect inversion method based on BP neural network and FEM is adopted in the following study. For indirect inversion method, many procedures such as selection of sample points, generation of sample FEM calculation file, FEM analysis, extraction of FEM results, generation of sample input and output files, establishment of mapping model, input of monitoring data, and output of the inversion parameters are involved. If the efficiency of carrying out these procedures cannot increase, more time might be required and the inversion efficiency might decrease. Consequently, the key to improve the efficiency of indirect conversion is to increase the automation level within and among the above procedures.

On the other hand, the inversion of mechanical parameters of surrounding rock, subsequent prediction, safety earlywarning, and revision of predesign are the critical procedures of feedback analysis during the construction of underground craven. At present, the data exchange among the above procedures is usually conducted by analyst [10]. However, in the essence, these procedures are related to each other and they constitute a continuous process, so the data exchange conducted by analyst leads to the decrease of the efficiency of feedback analysis. The key to solve this problem is to improve automation level of the connection of these procedures.

By considering the above mentioned, a method of intelligent autofeedback and safety early-warning for underground cavern is proposed based on BP neural network and FEM. The training sample point of BP neural network is selected by making use of uniform test design method, and autogeneration of FEM calculation file is realized by using the technique of file partition, information grouping, and orderly numbering. Then, the automation of FEM analysis, extraction of FEM results, generation of sample input and output files, inputting monitoring data and outputting inversion parameters, subsequent perdition, and safety early-warning are realized through programming. The software of intelligent autofeedback and safety early-warning for underground cavern engineering (IFWUC) during construction, which integrates the functions of intelligent inversion, subsequent prediction, and safety early-warning, is developed by taking ABAQUS as FEM analysis tool. Finally, the applicability of the proposed method and the developed software IFWUC is verified through an application example of underground cavern of a pumped-storage power station located in Southwest China.

\section{Selection of Sample Points of BP Neural Network}

In the study, BP neural network is adopted to establish the mapping model which can be used to simulate the relation of inversion parameters and monitoring data, and how to create sufficient training samples with statistic effects for network training is one of core issues for successful inversion [11].

At present, the methods used to choose the sample points for BP neural network are mainly orthogonal test design method, uniform test design method [11], and so forth. The uniform test design method is proposed based on orthogonal test design method and has been widely applied in practice. According to the principle of the number theory applied to multidimensional numerical integral, a set of uniform design tables, which can be taken as the basic tool for uniform test design, is created. In the study, uniform test design method is adopted to select sample points for it has the best representativeness on the premise of the same number of sample points.

In order to apply uniform test design method to choose sample points, the number of test factor and test design level should be given firstly. As to the inversion of mechanical parameters of surrounding rock, the number of test factors is that of inversion parameters, while the number of test design levels, which is used to determine the number of sample points (test times) combined with the selected uniform design table, should be chosen by considering practical requirements of the inversion problem. Once the value range of inversion parameters is given, sample points can be chosen according to the selected uniform design table. The entire training samples are considered as training sample set or training sample space. Each training sample is comprised of input part and output part. For the inversion of mechanics parameters of surrounding rock, the output part of a sample is the inversion parameters defined by the sample point, while the corresponding input part is the FEM results corresponding to monitoring data. As the displacement is chosen as the monitoring data in this study, the sample input is the displacement data extracted from FEM result file which should be obtained by conducting the analysis of the sample FEM calculation file. The whole sample input constitutes a sample input file, and the whole output constitutes a sample output file.

In order to increase the automation level of the selection of sample points, the automation of the sample point selection and the generation of the sample output file are achieved through programming.

\section{Autogeneration of FEM Calculation File for ABAQUS}

In order to obtain the displacement data needed by the generation of the sample input file, FEM analysis should be done for each FEM calculation file. As ABAQUS is taken as the FEM analysis tool in the study, the FEM calculation file should be generated according to the writing rules of ABAQUS. So the FEM calculation file is actually ABAQUS calculation file (hereafter referred to as INP file). As to each sample point, a sample INP file should be generated, and the entire sample INP files constitute a sample INP file set. In addition, prediction INP file used to realize the subsequent prediction should be also generated. For the deduction of 
work load of the generation of the sample INP file set and prediction INP file, the technique of file partition, information grouping, and orderly numbering is proposed based on the structure and writing rules of the ABAQUS calculation file.

The file partition, which is used to divide the INP file into several data blocks with different functions, is proposed based on the fixed structure of INP file. By making use of file partition, the INP files can be divided into several data blocks, which mainly are node definition data block, element definition data block, material grouping definition data block, boundary node setting definition data block, supporting structure information data block, excavation element setting definition data block, material parameters assignment data block, boundary conditions definition data block, and analysis step definition data block. These data blocks, which constitute the INP files, are automatically generated before the beginning of the optimization iteration and are not changed in the process of inversion.

The information grouping and orderly numbering are proposed for the purpose of being convenient to realize the autogeneration of INP file. For an underground cavern, the information involved in each data block is divided into several groups according to actual requirements, and then each group in any data blocks is orderly given a number starting from one. For example, supposing that the number of excavations of an underground cavern is set to $\mathrm{NN}$, the information of the excavation element setting definition data block can be divided into NN groups, and a common sign, which is a part of the name of the group, is given to all these groups. Then, each group orderly allocated a number from one to NN according to their actual order of excavation, and the name of each group is the combination of the common sign and the allocated number. Based on the information grouping and orderly numbering, the information of the INP file can be positioned and tracked accurately, and then the parametric autogeneration of INP file can be realized through programming.

At present, some software can be used to establish finite element model for underground craven, such as ABAQUS, ANSYS, and MARC. The FEM models established by different software have different formats of FEM calculation file, and different analysts are familiar with different software. As to the FEM model established by other software, corresponding INP file can be automatically generated by inputting required model data (such as node definition data, element definition data, and set definition data) into the developed software (see Section 6). Consequently, the compatibility between the developed software and other FEM software can be achieved.

\section{BPNN Intelligent Autoinversion of Mechanical Parameters}

Artificial neural network (ANN), which can be used to simulate certain mathematical mapping relation from input space to output space, is comprised of some artificial neurons with multi-input and single output. BP neural network

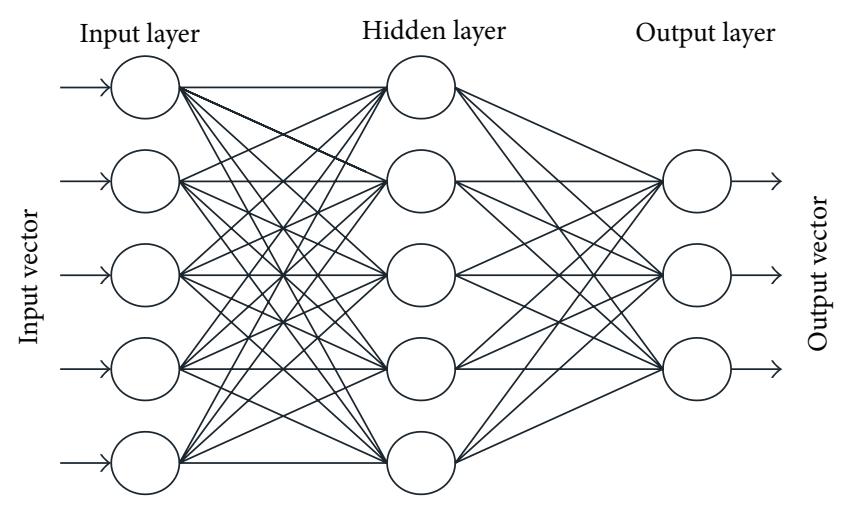

FIGURE 1: BP neural network model.

(BPNN), which adopts back propagation algorithm (BP algorithm) to train the network, has been applied in the inversion of mechanics parameters of surrounding rock [12, 13]. In general, BP neural network model includes input layer, hidden layer, and output layer (see Figure 1), each of which consists of a certain number of neurons (nodes).

In order to train the network, the training samples should be imported into the network through input layer, and then $\mathrm{BP}$ algorithm is used to adjust the link weight and deviation of the network with the aim of making network output approach the expected output. The training of network continues until the sum of square of the error of the network output is less than the error tolerance. Once the training is completed, the network can be used to simulate certain mapping relation.

Aiming at realizing BPNN (short for BP neural network) intelligent autoinversion of mechanics parameters of surrounding rock during construction, BPNN model should be trained by the using of the training samples, so the FEM analysis of sample INP file set should be accomplished before the creation of training samples. As the number of samples is generally large, it is inefficient and inconvenient to submit the INP file one by one. To solve this problem, the ABAQUS is automatically called for the FEM batch analysis through programming. The creation of the sample output file can be accomplished by extracting the displacement data of monitoring points from the FEM result files, and then the training samples can be given by combining the sample input file created in Section 2. During the creation of sample input file, the work load is usually heavy, especially when the numbers of monitoring points and sample points are large. In order to reduce the work load of creating the sample input file, a program module used to realize the autoextraction of the displacement data of monitoring points and autogeneration of sample input file is developed and integrated into IFWUC.

In addition, in order to realize BPNN intelligent autoinversion of mechanics parameters of surrounding rock during construction, the following issues should be also considered [10]: (1) the number of nodes of input layer and output layer; (2) the number of network layers; (3) the number of nodes of hidden layer; (4) original value of link weight; (5) excitation function of neuron.

The number of nodes of input and output layers depends on the number of dimensions of sample input and output vectors. The number of nodes of input layer is equal to 
the sum of displacement components of all monitoring points, while the number of nodes of output layer is equal to the number of inversion parameters.

The theoretical study of BP neural network reveals that two-layer network structure (one hidden layer and one output layer) can realize any nonlinear mapping. Although the increase of network layers can improve the precision, the efficiency might be decreased. Taking the inversion efficiency and inversion precision into account, BP neural network with two-layer network structure is adopted.

At present, there is no universal method of determination of the number of nodes of hidden layer, and it is usually determined by consideration of the efficiency and precision. The following formula is adopted to calculate the number of nodes of hidden layer:

$$
k=\sqrt{n+m}+a,
$$

where $n$ and $m$ are the number of nodes of input and output layers, respectively, and $a$ is the residual, and it is set to 15 according to the practical problem in this study.

Random number uniformly distributed among 0.1 0.9 is chosen for the original value of link weight according to the research results of $\mathrm{BP}$ algorithm.

The excitation functions of neurons, which are usually used to control the output of neuron, are mainly step function, sigmoid function, and so forth. The sigmoid function is used as excitation function of neuron due to its wide application; the expression of sigmoid function is given as follows:

$$
f(x)=\frac{1}{1+\exp (-b x)},
$$

where $b$ is slope of sigmoid function, which is usually set to 1 and $x$ is the input of excitation function.

According to $\mathrm{BP}$ algorithm, the autotraining program module of BP neural network is developed. Combined with the program module used to realize the autoextraction of the displacement data of monitoring points and autogeneration of sample input file, BPNN intelligent autoinversion of mechanical parameters of surrounding rock can be realized.

\section{Subsequent Prediction and Safety Early-Warning}

In addition to BPNN intelligent autoinversion of mechanical parameters of surrounding rock, subsequent prediction and safety early-warning based on the results of subsequent prediction are also required in order to accomplish feedback analysis.

The so-called subsequent prediction is to carry out the prediction of mechanical response (mainly represented by the deformation and stress) of surrounding rock and supporting structure according to subsequent construction steps (prediction steps) by making use of the parameters obtained from inversion. In order to realize the autoconnection of the two procedures of parameter inversion and subsequent prediction, a program module used to automatically perform two times of FEM analysis is developed and integrated into
TABLE 1: The allowable deformation.

\begin{tabular}{lccc}
\hline Rock type & \multicolumn{3}{c}{ Buried depth $(\mathrm{m})$} \\
& $<50$ & $50 \sim 300$ & $>300$ \\
\hline III & $0.10 \sim 0.30$ & $0.20 \sim 0.50$ & $0.40 \sim 1.20$ \\
IV & $0.15 \sim 0.50$ & $0.40 \sim 1.20$ & $0.80 \sim 2.00$ \\
V & $0.20 \sim 0.80$ & $0.60 \sim 1.60$ & $1.00 \sim 3.00$ \\
\hline
\end{tabular}

IFWUC. The first FEM analysis is done for the inversion steps using the parameters obtained from inversion, and then the second FEM analysis, which is used to obtain the FEM results of the prediction steps, is a restart analysis based on the FEM result file of the first FEM analysis by making use of the restart function of ABAQUS.

Based on subsequent prediction, safety early-warning for the stability of surrounding rock during construction can be conducted. The core issue for safety early-warning is the determination of early-warning indicator and earlywarning value. At present, there is not any unanimously recognized method of choosing early-warning indicator and early-warning value, and the stability of underground cavern is usually evaluated by considering the deformation, deformation velocity, deformation acceleration, distribution of plastic zone, the working status of supporting structure, and so forth.

As deformation is considered as the basic information which can be used to evaluate whether surrounding rock is stable or not and most of the related specifications take the allowable deformation as the indicator to evaluate the stability of surrounding rock, the deformation of surrounding rock is adopted as early-warning indicator in this paper.

As the deformation of surrounding rock of underground cavern during construction is affected by several factors including geological conditions and craven scale, allowable deformation (early-warning value) should be different for different underground caverns. In view of this, two methods are provided to determine allowable deformation in the developed safety early-warning program, one of which is the allowable deformation automatically calculated by the program according to the corresponding regulation (shown in Table 1) in Chinese Technical Specification of Shotcrete and Rock Bolt for Water Resources and Hydropower Project (SL377-2007) and the other of which is the analyst which directly defines the allowable deformation in the program.

Based on the aforementioned method, a program module, which can realize the autoextraction of deformation at early-warning control points (defined by analyst), the following autocomparison with allowable deformation, and autooutput of early-warning information, is developed and integrated into IFWUC. Consequently, the autoconnection of the two procedures of subsequent forecast and safety earlywarning can be achieved.

\section{Analysis Procedures and Software Development}

The intelligent autofeedback and safety early-warning procedures are shown in Figure 2. 


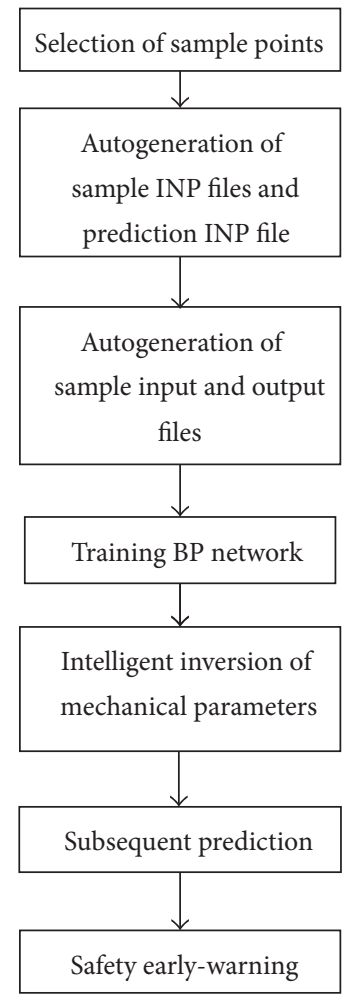

FIGURE 2: The procedures of feedback analysis.

The software of intelligent autofeedback and safety earlywarning for underground cavern engineering during construction (IFWUC), which is integrated by the modules of parameter inversion, subsequent prediction, and safety early-warning, is developed on the platform of VISUAL STUDIO 2008 and by making use of the mixed programming technique of FORTRAN and $\mathrm{C}++$. ABAQUS is taken as the FEM analysis tool of IFWUC and is automatically called by IFWUC if needed.

IFWUC is developed with several features, and the main features are listed in the following:

(1) inversing the deformation parameters and strength parameters separately, or both of them,

(2) considering the ordinary supporting structures in the version if needed,

(3) being adaptive to both 2D and 3D problems,

(4) inversing mechanical parameters based on either the point displacement measured by the multipoint extensometer or the convergence displacement measured by the convergence gauge,

(5) being able to automatically perform subsequent prediction and safety early-warning.

The main advantages of IFWUC are that it can realize the intelligent autoinversion of mechanical parameters of surrounding rock and the autoconnection of parameters inversion, subsequent prediction, and safety early-warning. These advantages can increase the efficiency of the feedback

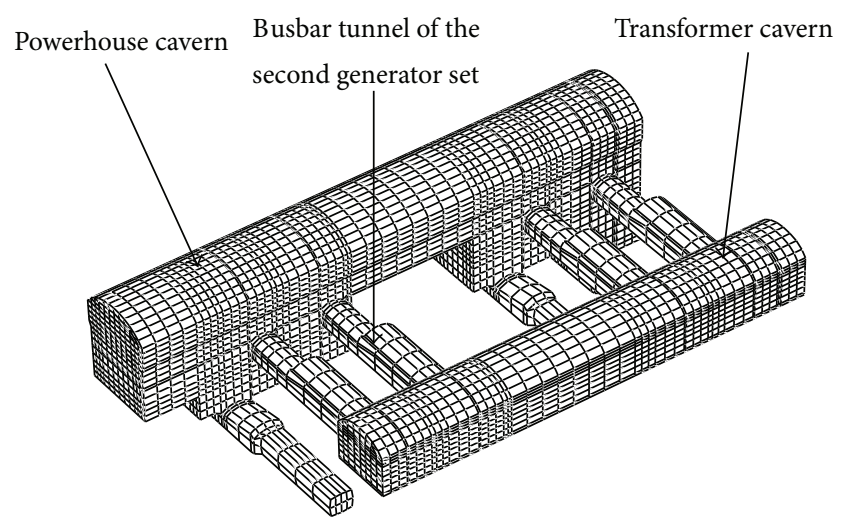

Figure 3: 3D model.

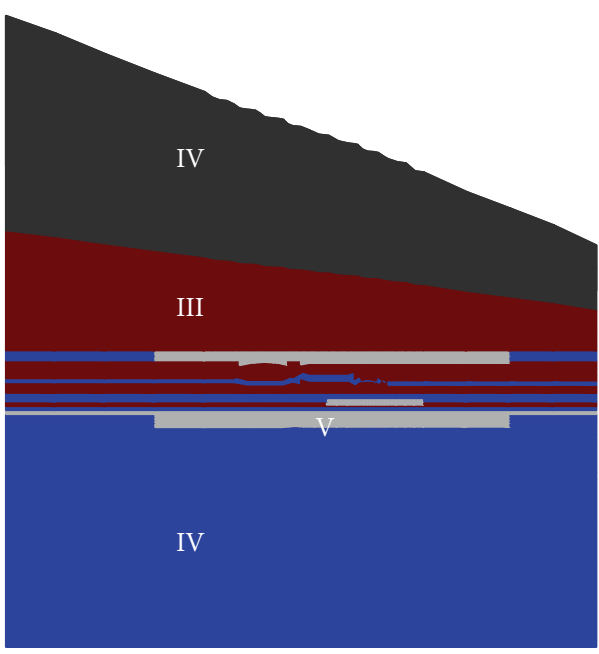

FIgURE 4: The region of different rock types.

analysis of underground craven engineering and reduce the difficulty of its application in practical engineering.

\section{An Application Example}

The underground caverns of a pumped-storage power station located in Southwest China are composed of waterconveyance tunnel, powerhouse cavern, transformer cavern, busbar tunnel, transportation tunnel, and other construction branch caverns. The 3D FEM mesh of powerhouse cavern, transformer cavern, tailrace tunnels, and busbar tunnel is shown in Figure 3.

In the study, the powerhouse cavern, transformer cavern, and busbar tunnel are considered, and the symmetrical section of busbar tunnel of the second generator set is chosen for feedback analysis. The rock type of the section is given in Figure 4.

Figure 5 shows the section's FEM model, which is mainly composed of the four-node isoparametric element. In FEM model, the positive direction of $x$-axis is vertical to the longitudinal section of the powerhouse cavern and points to the downstream, while the positive direction of $y$-axis is upward and perpendicular to the horizontal plane. The width 
TABLE 2: The design value of physical and mechanical parameters.

\begin{tabular}{lccccc}
\hline Rock type & Young's modulus $(\mathrm{GPa})$ & Poisson ratio & Cohesion $(\mathrm{MPa})$ & Friction degree $\left(^{\circ}\right)$ & Density $\left(\mathrm{kg} / \mathrm{m}^{3}\right)$ \\
\hline III & 6.5 & 0.21 & 0.95 & 43.4 & 2500 \\
IV & 4.5 & 0.23 & 0.60 & 36.5 & 2520 \\
V & 1.5 & 0.25 & 0.25 & 24.2 & 2610 \\
\hline
\end{tabular}

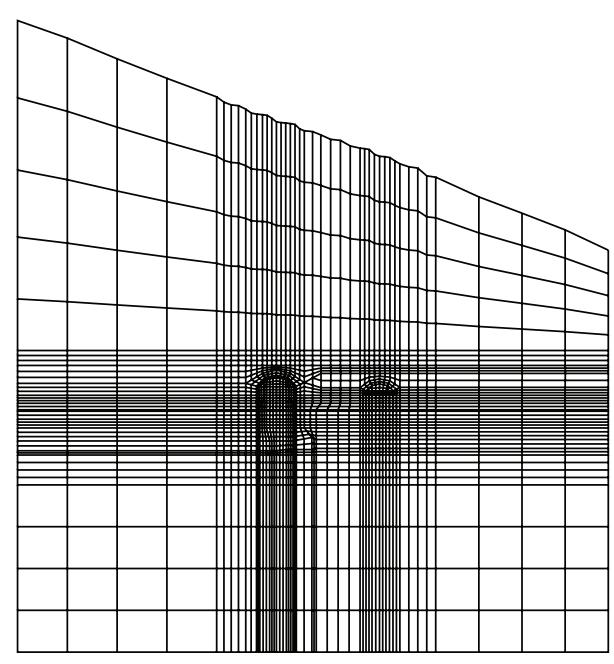

FIgure 5: The FEM model of the section chosen for studying.

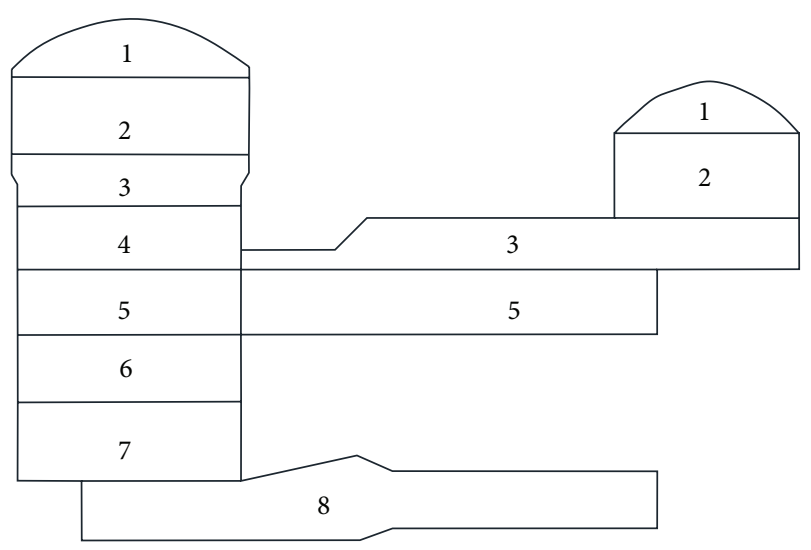

Figure 6: Excavation scope of the underground caverns.

of the model is set to $455 \mathrm{~m}$, which is big enough for considering the effect of excavation. The bottom of the model is set $130 \mathrm{~m}$ below the bottom of tailrace tunnels, and the top of the model is up to the ground surface.

In FEM analysis, the lateral and bottom of the model are fixed in normal direction, while the top of the model is free. The initial geostress field of the model is taken as the gravity stress field based on the real geological condition, and the Drucker-Prager criterion is adopted as the yield criterion of surrounding rock. The number of excavation steps is 8 , and the excavation scope of each step is shown in Figure 6. The design value of physical and mechanical parameters of surrounding rock is given in Table 2 .

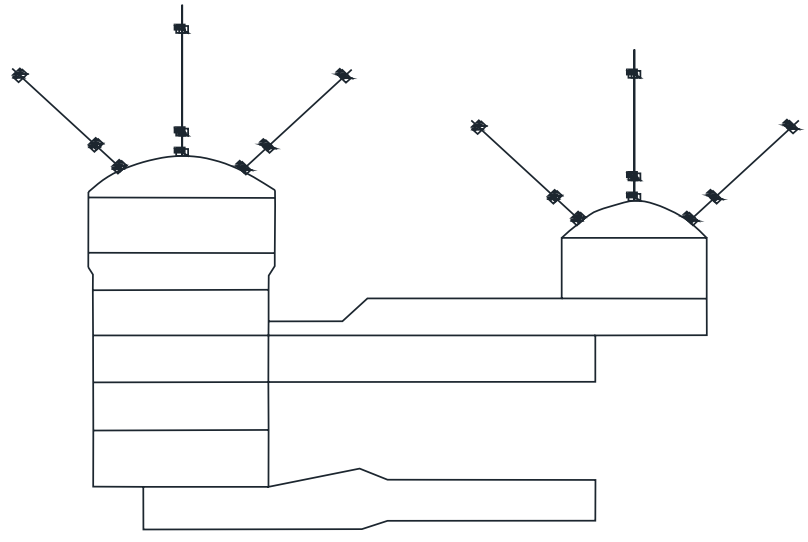

FIGURE 7: Layout of the measuring points.

Since the excavation of the underground caverns has not started, the monitoring data is not available at present. In order to proceed with the application of the software developed in Section 6 to this project, the displacements of the monitoring points, which are calculated using FEM method under the design value of the mechanical parameters, are taken as the monitoring data required by feedback analysis. Figure 7 gives the layout of the monitoring points, and the feedback analysis, which includes parameter inversion, subsequent prediction, and safety early-warning, is assumed to be done after the first step of excavation.

The convergence deformation of several pairs of characteristic points of the excavation section is chosen as the early-warning indicator, and the allowable deformation is automatically calculated by the software according to Table 1 given in Chinese Technical Specification of Shotcrete and Rock Bolt for Water Resources and Hydropower Project (SL377-2007). Two pairs of characteristic points are set on the excavation section of powerhouse cavern. The first pair is comprised of the arch center and bottom center, and the second pair is comprised of the left arch foot and the right arch foot. The other two pairs of characteristic points are set on the excavation section of transformer cavern, and the first and second pairs are comprised of the arch center and bottom center, the left arch foot and the right arch foot, respectively.

In order to get precise inversion results, the inversion parameters (namely, test factors) should be sensitive to the monitoring data. Therefore, considering the surrounding rock concerned in excavation step 1 mainly belongs to types III and IV (shown in Figure 4), the inversion parameters are chosen as Young's modulus of types III and IV, and the value of other parameters is taken as their design value listed in Table 2. In the process of inversion, the range of 


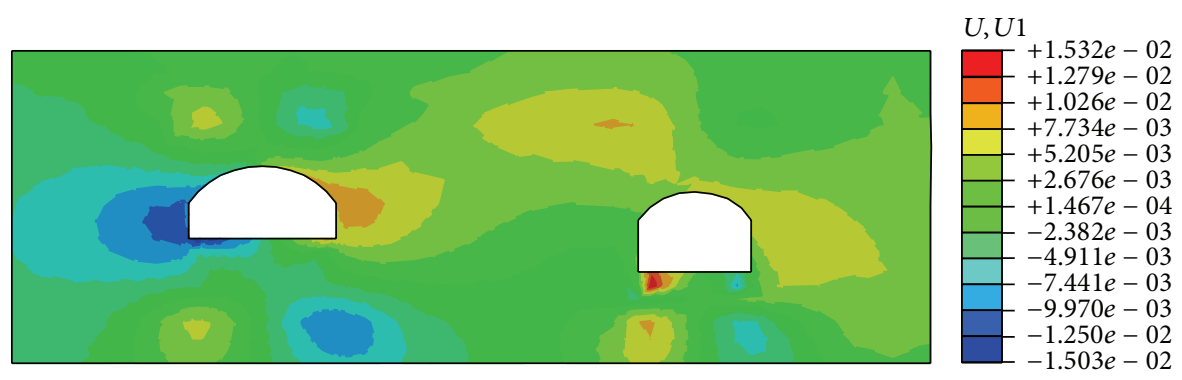

FIgURE 8: The contour of horizontal displacement (m).
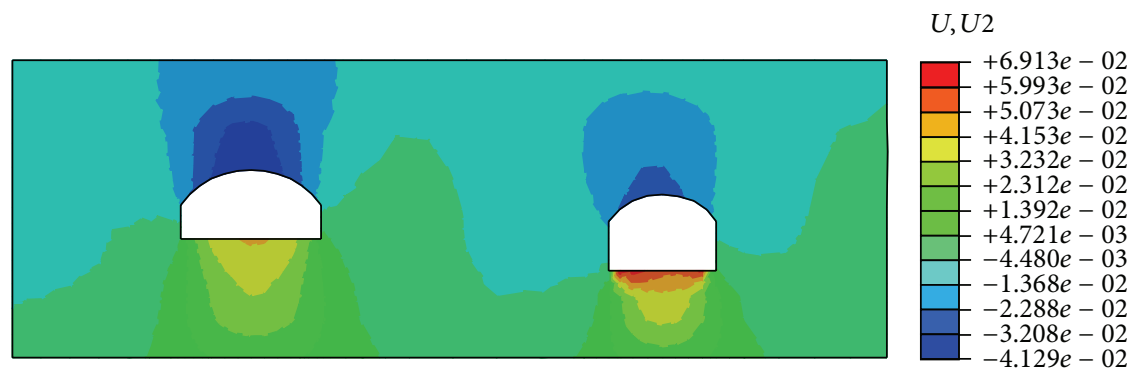

Figure 9: The contour of vertical displacement (m).

TABLE 3: The design and inversion value of parameters.

\begin{tabular}{lcc}
\hline Rock type & $\begin{array}{c}\text { Young's modulus/GPa } \\
\text { (inversion value) }\end{array}$ & $\begin{array}{c}\text { Young's modulus/GPa } \\
\text { (design value) }\end{array}$ \\
\hline III & 6.49 & 6.5 \\
IV & 4.47 & 4.5 \\
\hline
\end{tabular}

inversion parameters is limited between 1.0 GPa and 10.0 GPa according to the geological survey, and the number of sample points is set to 10 .

The inversion value of Young's modulus of rock types III and IV is listed in Table 3, and the displacements distribution of surrounding rock obtained from subsequent prediction is shown in Figures 8 and 9.

From Table 3, it can be found that the inversion value obtained by BPNN intelligent inversion is quite close to the design value. From Figures 8 and 9, it is shown that the subsequent prediction is successfully conducted based on the parameter inversion.

The results of automatic safety early-warning are given in Table 4. From Table 4, it can be found that the vertical convergence deformation of both powerhouse cavern and transformer cavern exceeds the allowable deformation calculated by IFWUC, which means the surrounding rock is unstable if the excavation of step 2 is conducted without taking supporting measures after the excavation of step 1 . In order to ensure the stability of surrounding rock during construction, necessary supporting measures should be applied to surrounding rock after the excavation of step 1.

From the aforementioned discussion, it can be concluded that the proposed method and the developed software
IFWUC can be applied to practical underground engineering.

\section{Conclusions}

In order to solve the existing problems of the low efficiency of parameter inversion and the fail to realize automatic exchange of data among parameter inversion, subsequent prediction, and early-warning in the process of feedback analysis of underground cavern engineering, the method of intelligent autofeedback and safety early-warning is proposed based on uniform test design method, BP neural network, and FEM, and the corresponding software is well developed. The applicability of the proposed method and the developed software IFWUC is verified through an application example of underground cavern of a pumped-storage power station located in Southwest China.

The feedback analysis of underground craven during construction is a dynamic process interrelated and paralleled with the construction at site. In this field, some problems, such as the uniqueness of inversion parameters, dynamic evolution of mechanics properties of surrounding rock during construction, and early-warning indicator with better representativeness, still need further research in the future.

\section{Conflict of Interests}

The authors declare that there is no conflict of interests regarding the publication of this paper. 
TABLE 4: The results of safety early-warning.

\begin{tabular}{lcccc}
\hline & $\begin{array}{c}\text { Pairs of } \\
\text { characteristic } \\
\text { points }\end{array}$ & $\begin{array}{c}\text { Convergence } \\
\text { deformation } \\
(\mathrm{cm})\end{array}$ & $\begin{array}{c}\text { Allowable } \\
\text { deformation } \\
(\mathrm{cm})\end{array}$ & 7.8 \\
$\begin{array}{l}\text { Powerhouse } \\
\text { cavern }\end{array}$ & $\begin{array}{c}\text { Arch center } \\
\text { Bottom center } \\
\text { Left arch foot } \\
\text { Right arch foot }\end{array}$ & 8.0 & 15.9 & Safety early-warning \\
\hline $\begin{array}{l}\text { Transformer } \\
\text { cavern }\end{array}$ & $\begin{array}{c}\text { Arch center } \\
\text { Bottom center } \\
\text { Left arch foot } \\
\text { Right arch foot }\end{array}$ & 10.1 & 8.6 & Safe \\
\hline
\end{tabular}

\section{Acknowledgment}

This work was supported by projects of the National Natural Science Foundation of China (Grant nos. 51109067, 11132003).

\section{References}

[1] Z. Li, Z. Zhou, Q. Xu et al., "Implementation and development of AI back analysis for the underground powerhouse of Jinping-1 hydropower project," Chinese Journal of Hydroelectric Engineering, vol. 29, no. 3, pp. 177-183, 2010.

[2] R. R. Han, J. H. Zhang, X. Zhang et al., "Study of prediction on feedback calculation of excavated monitor to the underground powerhouse of Xiluodu," Chinese Journal of Shandong University (Engineering Science), vol. 39, no. 4, pp. 140-144, 2009.

[3] G. Wang, Y. J. Jiang, C. S. Li et al., "Rapid feedback analysis method for underground caverns during construction," Chinese Journal of Shandong University (Engineering Science), vol. 41, no. 8, pp. 133-136, 2011.

[4] S.-H. Chen, S.-F. Chen, I. Shahrour, and P. Egger, "The feedback analysis of excavated rock slope," Rock Mechanics and Rock Engineering, vol. 34, no. 1, pp. 39-56, 2001.

[5] X. Yao, N. Li, L. Chen, H. Sun, and M. Jing, "Back analysis of surrounding rock stability based on excavation process of underground powerhouse at Laxiwa hydropower station," Chinese Journal of Rock Mechanics and Engineering, vol. 30, no. S1, pp. 3052-3059, 2011.

[6] S.-P. Jia, G.-J. Wu, and W.-Z. Chen, "Application of finite element inverse model based on improved particle swarm optimization and mixed penalty function," Rock and Soil Mechanics, vol. 32, no. 2, pp. 598-603, 2011.

[7] Q. Jiang, X. Feng, G. Su, and G. Chen, "Intelligent back analysis of rock mass parameters for large underground caverns under high earth stress based on EDZ and increment displacement," Chinese Journal of Rock Mechanics and Engineering, vol. 26, no. S1, pp. 2654-2662, 2007.

[8] L. Xu and T.-J. Zhang, "Development of automatic system for inversion of mechanical parameters and advanced prediction of mechanical response of surrounding rock of underground cavern in the period of construction," Journal of Sichuan University (Engineering Science Edition), vol. 45, no. 6, pp. 5157, 2013.

[9] D. Yi, S. H. Chen, and X. R. Ge, "A methodology combining genetic algorithm and finite element method for back analysis of initial stress field of rock masses," Chinese Journal of Rock and Soil Mechanics, vol. 32, supplement 2, pp. 598-603, 2011.
[10] K. Guo, Research and implementation of feedback analysis system for underground group caverns in construction-period [Dissertation], Tsinghua University, Beijing, China, 2010.

[11] H. Tang, G. Shi, X. Ge, X. Xiang et al., "Application of uniform design to displacement back analysis in underground engineering," Chinese Journal of Rock Mechanics and Engineering, vol. 25, no. 10, pp. 2017-2022, 2006.

[12] W. P. Nie, Y. H. Yan, C. Shi et al., "Inversion and feedback analysis of mechanical parameters of surrounding rock of relief well of Nuozhadu hydropower station," Chinese Journal of Hohai University (Natural Sciences), vol. 39, no. 1, pp. 54-60, 2011.

[13] C.-H. Fu, W.-X. Qin, S.-H. Chen, and H.-B. Zhou, "Development and application of rock-mass mechanical parameters back analysis software system for underground cavern," Rock and Soil Mechanics, vol. 28, no. 3, pp. 577-581, 2007. 


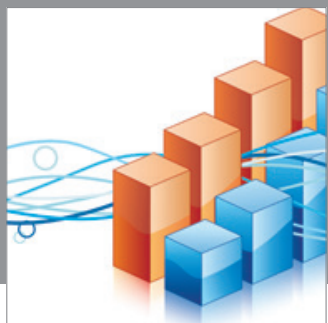

Advances in

Operations Research

mansans

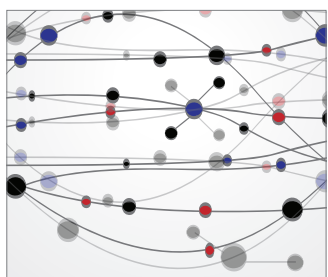

The Scientific World Journal
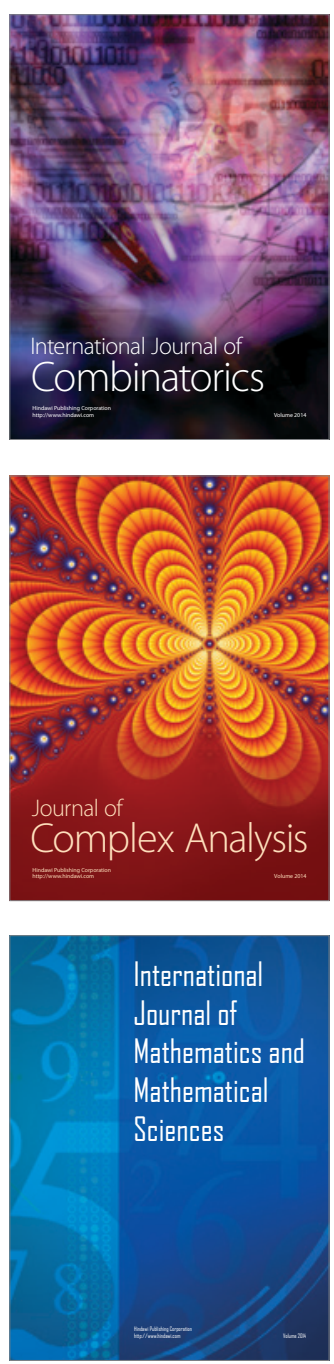
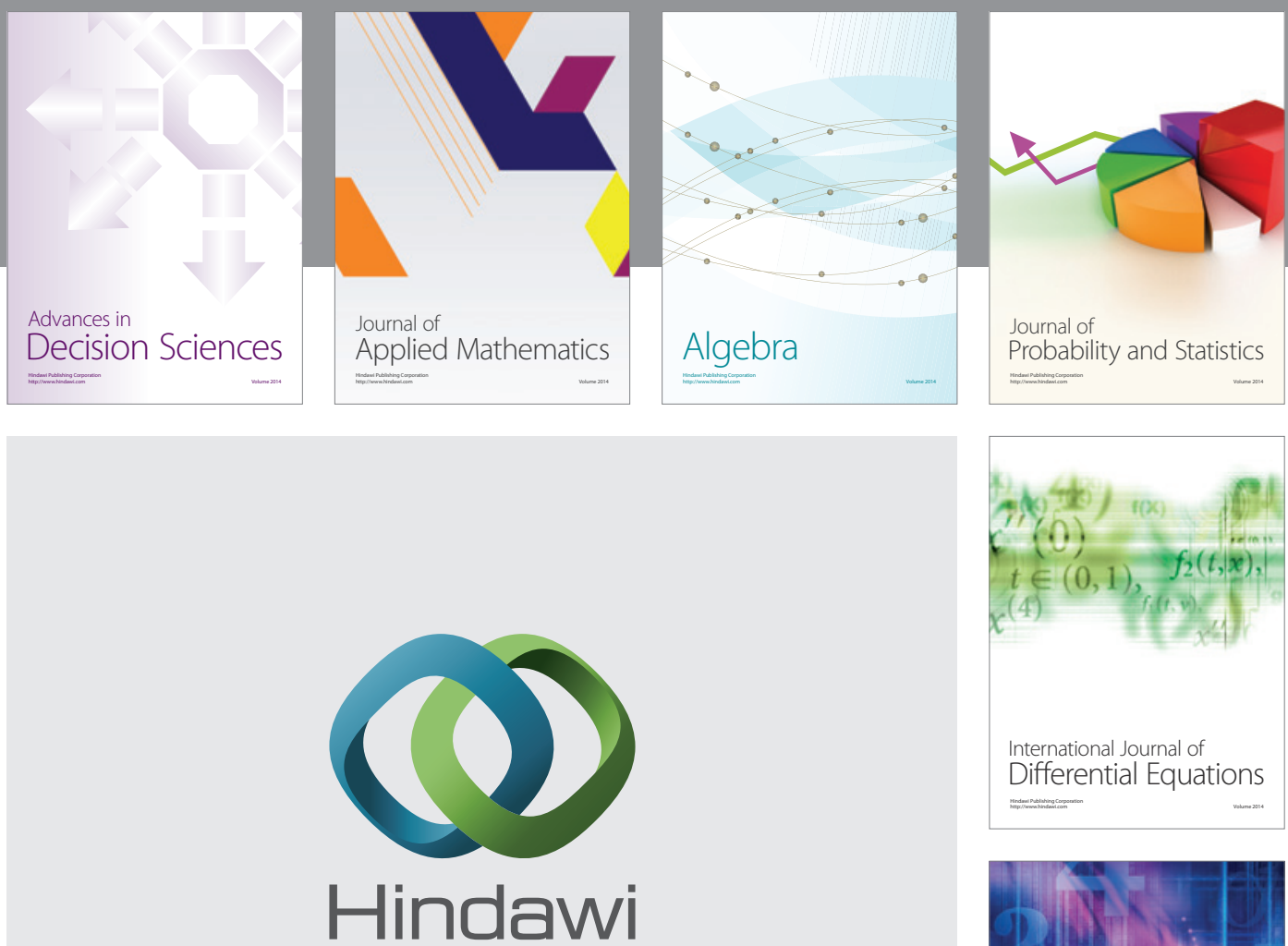

Submit your manuscripts at http://www.hindawi.com
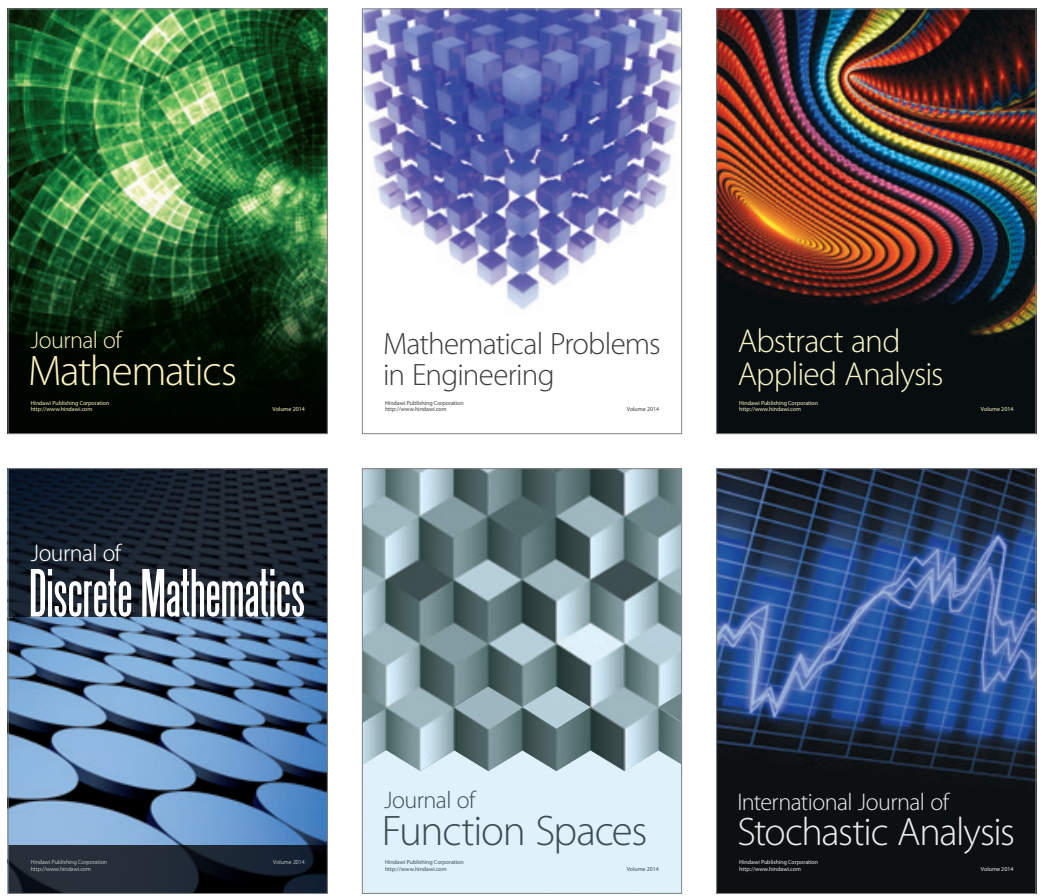

Journal of

Function Spaces

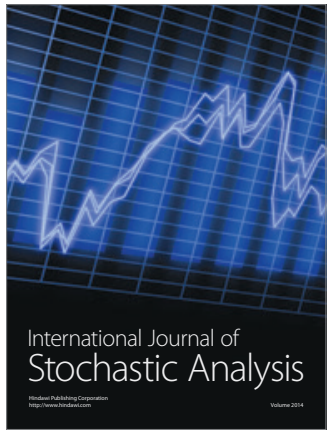

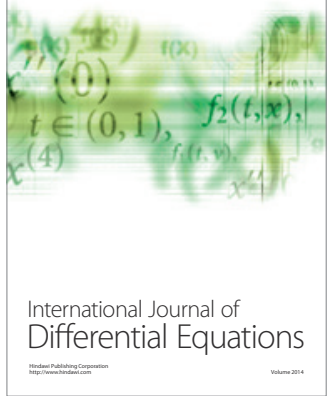
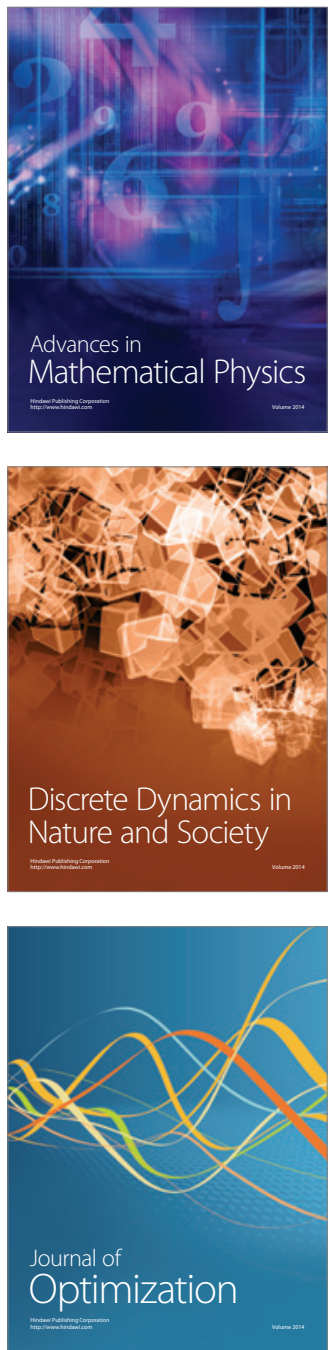